\title{
First-Principles Approach to Nonlinear Lattice Dynamics: Anomalous Spectra in PbTe
}

\author{
Yue Chen (陈粤), ${ }^{1 *}$ Xinyuan Ai (艾馨元) ${ }^{2}$ and C. A. Marianetti ${ }^{1}$ \\ ${ }^{1}$ Department of Applied Physics and Applied Mathematics, Columbia University, New York, New York 10027, USA \\ ${ }^{2}$ Department of Physics, Columbia University, New York, New York 10027, USA
}

(Received 12 January 2014; published 2 September 2014)

\begin{abstract}
Here we introduce a new approach to compute the finite temperature lattice dynamics from first principles via the newly developed slave mode expansion. We study $\mathrm{PbTe}$ where inelastic neutron scattering reveals strong signatures of nonlinearity as evidenced by anomalous features which emerge in the phonon spectra at finite temperature. Using our slave mode expansion in the classical limit, we compute the vibrational spectra and show remarkable agreement with temperature dependent inelastic neutron scattering measurements. Furthermore, we resolve an experimental controversy by showing that there are no appreciable local nor global spontaneously broken symmetries at finite temperature and that the anomalous spectral features simply arise from two anharmonic interactions. Our approach should be broadly applicable across the periodic table.
\end{abstract}

DOI: 10.1103/PhysRevLett.113.105501

PACS numbers: 63.20.Ry, 63.20.dk, 84.60.Rb

Inelastic neutron scattering (INS) is a fundamental probe of materials which has allowed a unique view into the most basic aspects of mechanical behavior [1]. With the advent of the Spallation Neutron Source, massive amounts of data can be accumulated at an unprecedented rate, allowing for an extremely detailed inspection of phenomena throughout reciprocal space. Despite the enormous success of this experimental method, theory has greatly lagged behind in the context of vibrations. At present, one cannot compute the temperature dependence of the phonon spectrum for materials with appreciable phonon interactions from first principles. Moreover, this scenario cannot even be routinely handled in the classical limit for indirect reasons. This assertion is empirically well illustrated by the strongly interacting phonon material $\mathrm{PbTe}$. INS measurements demonstrated signatures of strong interactions in the temperature dependence of the phonon spectra [2,3], attracting significant attention to this system, yet complementary theoretical predictions have not yet been made. In this Letter, we circumvent previous theoretical limitations, and resolve the experimental anomalies in PbTe.

In many materials, density functional theory (DFT) is expected to describe the structural energetics to a high degree of accuracy. Therefore, the interatomic potential generated via solving the DFT equations (assuming the Born-Oppenheimer approximation) should be reliable to perform both quantum and classical dynamics of the nuclei. However, the problem is that the scaling of DFT is sufficiently prohibitive to forbid this in many scenarios. Even at the level of classical mechanics (also known as $a b$ initio molecular dynamics), one would need a very large unit cell along with many time steps. In most cases this prevents one from directly being able to compute even the classical vibrational spectrum at finite temperature, as evidenced by the sparse number of such publications in the literature. There are a variety of approaches that attempt to circumvent this problem. One approach is the use of an empirical potential in place of DFT. This is acceptable if an accurate empirical potential exists, but this is generally not the case. Another approach would be in the spirit of static mean-field theories based on DFT energetics. Two such approaches are the self-consistent $a b$ initio lattice dynamics (SCAILD) method [4] and the approach of Hellman et al. [5], both of which have been used to capture the static phonon renormalization as a function of temperature. The well-known quasiharmonic approximation has various limitations while often being less reliable than the aforementioned approaches [6]. Another avenue is using more efficient electronic structure methods. Tight-binding based approaches [7] typically have excessive errors even at the level of phonon frequencies. Linear-scaling DFT approaches [8,9] have definitely bridged the length scale, but the time scale is still a formidable barrier to obtaining a robust spectra, in addition to the open questions regarding the precision of the basis set. A final approach would be to Taylor series expand the DFT energies as a function of the atomic displacements (see Ref. [10] for a detailed overview). This would retain all the accuracy of DFT to a certain range and order, while having a relatively negligible cost:

$$
V=V_{H}+\sum_{i j k} \Psi_{i j k} u_{i} u_{j} u_{k}+\sum_{i j k l} \chi_{i j k l} u_{i} u_{j} u_{k} u_{l}+\cdots,
$$

where $V_{H}$ is the harmonic contribution; $\Psi$ and $\chi$ denote the third and fourth order force constants, respectively; $u$ is the atomic displacement, while indices $i, j, k$, and $l$ label both the atom and the cartesian coordinate. There is a long history of performing such an expansion in various degrees 
of sophistication, and this approach and the details of all the nontrivial symmetries that must be satisfied have been well outlined [10]. However, the very few demonstrably robust executions of this straightforward Taylor series highlight the difficulties of computing many expansion coefficients while simultaneously satisfying all the symmetries. A Taylor series to third order and within nearest neighbor was used in $\mathrm{PbTe}$ [11], and while this did reproduce the thermal conductivity, the spectra was not computed. Recently, we have introduced a new approach called the slave mode expansion [12], which essentially makes the Taylor series substantially more tractable as evidenced by our ability to straightforwardly compute all 358 anharmonic expansion coefficients which exist within an octahedra (i.e., up to next-nearest neighbor interactions) up to fourth order. In this Letter, we demonstrate the utility of our approach by computing classical vibrational spectra for $\mathrm{PbTe}$, resolving the anomalous behavior in $\mathrm{PbTe}$ and ending experimental discrepancy.

$\mathrm{PbTe}$ has garnered significant interest as a thermoelectric material [13]. Bate et al. [14] found that the undistorted ground state structure of $\mathrm{PbTe}$ transforms to a paraelectric phase at elevated temperatures, which contradicts the conventional picture of ferroelectric-paraelectric transformation. More recently, Božin et al. [6] proposed that the stable local dipoles emerge from the undistorted structure in $\mathrm{PbTe}$ based on the analysis of the pair distribution function. In addition, the phonon dispersions in $\mathrm{PbTe}$ were recently measured using INS at different temperatures $[2,3]$. It was found that the phonon scattering in $\mathrm{PbTe}$ is strongly anharmonic and displays anomalous behavior at elevated temperatures, i.e., the "waterfall" effect of the transverse optical (TO) phonon mode at $\Gamma$ point and the avoided-crossing behavior between the longitude acoustic and TO branches in the $\Gamma \rightarrow X$ direction. More importantly, a new spectral feature emerges at the zone center at finite temperature, which is a clear signature of strong interactions and requires a theoretical explanation.

Theory has already made a number of contributions in understanding PbTe. The phonon dispersions have been studied in numerous publications by using either the finite displacement $[11,15]$ or linear response methods [16-18] based on DFT. The SCAILD method successfully captured the qualitative trend for the shift of the TO-mode spectra to higher frequency with increasing temperature [6], while the quasiharmonic approximation failed by predicting the opposite [16]. However, the preceding study was incapable of predicting a redistribution of spectral weight and therefore it cannot capture the emergence of the anomalous spectra at the zone center (i.e., it only shifts the delta function without any broadening). Ab initio molecular dynamics (AIMD) simulations reproduced the nonGaussian asymmetric behaviors of the pair distribution function peaks in PbTe [19]; however, because of the limited number of atoms and short time scale that AIMD can currently simulate, the vibrational spectra cannot be effectively evaluated from the trajectories. In addition, empirical interatomic potentials, which have been developed for PbTe [20,21], fail to capture the most important features of the phonon dispersions, i.e., an extremely soft TO mode near the $\Gamma$ point. Therefore, none of the existing techniques can handle strongly incoherent behavior demonstrated in $\mathrm{PbTe}$, where at certain wave vectors the concept of a mode lifetime is rendered meaningless.

In order to compute the vibrational spectra from DFT forces, we have used the slave mode expansion [12] to construct a polynomial interatomic potential containing all terms within next-nearest neighbors and up to fourth order for $\mathrm{PbTe}$. We show that there are 56 terms at third order and 302 at fourth order, and this is dictated purely by symmetry. Density functional theory has been used to compute all 358 terms, and we have demonstrated that these have a high fidelity and that longer range interactions are small [12]. Moreover, we have proven that one can perform a unitary transformation to construct a minimal model of anharmonicity which captures the vast majority of the physics with simply one term at third order and one term at fourth order. The resulting minimal potential can be compactly expressed as follows:

$$
\begin{aligned}
V= & V_{H} \\
& +\Phi_{3} \sum_{\mathbf{R}}\left(\phi_{\mathbf{R} x_{-}}^{3}-\phi_{\mathbf{R} x_{+}}^{3}+\phi_{\mathbf{R} y_{-}}^{3}-\phi_{\mathbf{R} y_{+}}^{3}+\phi_{\mathbf{R} z_{-}}^{3}-\phi_{\mathbf{R} z_{+}}^{3}\right) \\
& +\Phi_{4} \sum_{\mathbf{R}}\left(\phi_{\mathbf{R} x_{-}}^{4}+\phi_{\mathbf{R} x_{+}}^{4}+\phi_{\mathbf{R} y_{-}}^{4}+\phi_{\mathbf{R} y_{+}}^{4}+\phi_{\mathbf{R} z_{-}}^{4}+\phi_{\mathbf{R} z_{+}}^{4}\right)
\end{aligned}
$$

and

$$
\begin{aligned}
& \phi_{\mathbf{R} z_{-}}=\frac{1}{\sqrt{2}}\left(u_{T e, z}^{\mathbf{R}+\mathbf{a}_{1}}-u_{P b, z}^{\mathbf{R}}\right), \quad \phi_{\mathbf{R} z_{+}}=\frac{1}{\sqrt{2}}\left(u_{T e, z}^{\mathbf{R}+\mathbf{a}_{2}+\mathbf{a}_{3}}-u_{P b, z}^{\mathbf{R}}\right), \\
& \phi_{\mathbf{R} x_{-}}=\frac{1}{\sqrt{2}}\left(u_{T e, x}^{\mathbf{R}+\mathbf{a}_{2}}-u_{P b, x}^{\mathbf{R}}\right), \quad \phi_{\mathbf{R} x_{+}}=\frac{1}{\sqrt{2}}\left(u_{T e, x}^{\mathbf{R}+\mathbf{a}_{1}+\mathbf{a}_{3}}-u_{P b, x}^{\mathbf{R}}\right), \\
& \phi_{\mathbf{R} y_{-}}=\frac{1}{\sqrt{2}}\left(u_{T e, y}^{\mathbf{R}+\mathbf{a}_{3}}-u_{P b, y}^{\mathbf{R}}\right), \quad \phi_{\mathbf{R} y_{+}}=\frac{1}{\sqrt{2}}\left(u_{T e, y}^{\mathbf{R}+\mathbf{a}_{1}+\mathbf{a}_{2}}-u_{P b, y}^{\mathbf{R}}\right),
\end{aligned}
$$

where $\phi$ are the slave modes which are simply dimers in this case, and $\mathbf{a}_{\mathbf{i}}$ are the primitive lattice vectors of PbTe: $\mathbf{a}_{1}=a / 2(1,1,0), \quad \mathbf{a}_{2}=a / 2(0,1,1), \quad$ and $\mathbf{a}_{3}=$ $a / 2(1,0,1)$. There are six dimer slave modes per primitive unit cell, one corresponding to each $\mathrm{Pb}$-Te octahedral bond, and these are simply a displacement difference between corresponding vectors of $\mathrm{Pb}$ and Te. The values for the expansion coefficients are found to be $\Phi_{3}=2.68 \mathrm{eV} / \AA^{3}$ and $\Phi_{4}=3.70 \mathrm{eV} / \AA^{4}$, respectively. These parameters were computed without spin-orbit coupling, and this is known to overpredict the $T=0 \mathrm{~K}$ TO-mode phonon frequency [17], which should be accounted for when comparing to experiments. 
Classical dynamics simulations are performed using Eq. (2). A $10 \times 10 \times 10$ supercell of the conventional unit cell containing 8000 atoms is used in the simulations with periodic boundary conditions. The microcanonical ensemble and a time step of 5 fs are applied. The system is initialized with random atomic displacements and equilibrated for $50 \mathrm{ps}$. The atomic velocities and positions are collected every 20 time steps in the following $1 \mathrm{~ns}$. Using the Wiener-Khintchine theorem [22], we can compute the power spectra $Z_{q}(\omega)$ at a given wave vector $q$ given the trajectory:

$$
Z_{q}(\omega)=\frac{1}{2 \pi} \sum_{\alpha, s} \frac{m_{\alpha}}{N_{q}}\left|\int d t e^{-i \omega t} \sum_{j} v_{j}^{(\alpha, s)}(t) e^{-i q \cdot r_{j}^{\alpha}(t)}\right|^{2},
$$

where $v$ is the velocity, $r$ is the position, $\alpha$ labels the atom type (i.e., $\mathrm{Pb}$ or $\mathrm{Te}$ ), $j$ labels a particular atom within a given type, $s$ labels the displacement vector (i.e., $x, y, z$ ), and $N_{q}$ is the number of $q$ points. The power spectrum is related to the imaginary part of the phonon Green's function in the classical limit, and this serves as a proxy for what is measured in INS experiments.

The finite supercell size of 4000 primitive units in our MD simulations yields 4000 wave vectors as a discrete grid over the Brillouin zone. Figure 1 shows the spectra along selected high symmetry lines in the first Brillouin zone. At finite temperatures, the phonons will interact and no longer exist at a well-defined frequency. The most elementary many-body renormalizations are broadening (i.e., diminished lifetime) and shifting of the peaks, both of which can be seen within perturbation theory [22]. The white curves are the phonon dispersions (i.e., zero temperature) of Zhang et al. [15], and only mild renormalizations are observed at the relatively low temperature of $T=13 \mathrm{~K}$. However, it is already evident that the acoustic modes are much less affected by the interactions as compared to the optical modes.

At $T=380 \mathrm{~K}$, much more pronounced effects are observed. The low energy acoustic modes still show relatively mild broadening and shifts, while other branches have substantially shifted and broadened. The most interesting behavior is observed at the zone center where the spectrum has remarkably split into two broad peaks, as measured in experiments [2,3]. Additionally, the whole branch of TO phonons shift significantly to higher frequencies at this temperature, which explains the striking experimental observation of the avoided crossing between the TO and longitude acoustic branches in the $\Gamma \rightarrow X$ direction.

In order to further elucidate the behavior at the zone center, the $q=0$ spectrum is computed at a range of temperatures [see Fig. 2(a)]. At the lowest temperature $T=13 \mathrm{~K}$ the peak is sharp, while signatures of nonlinearities can clearly be seen at $T=54 \mathrm{~K}$ with the formation of a second feature at slightly higher energy.
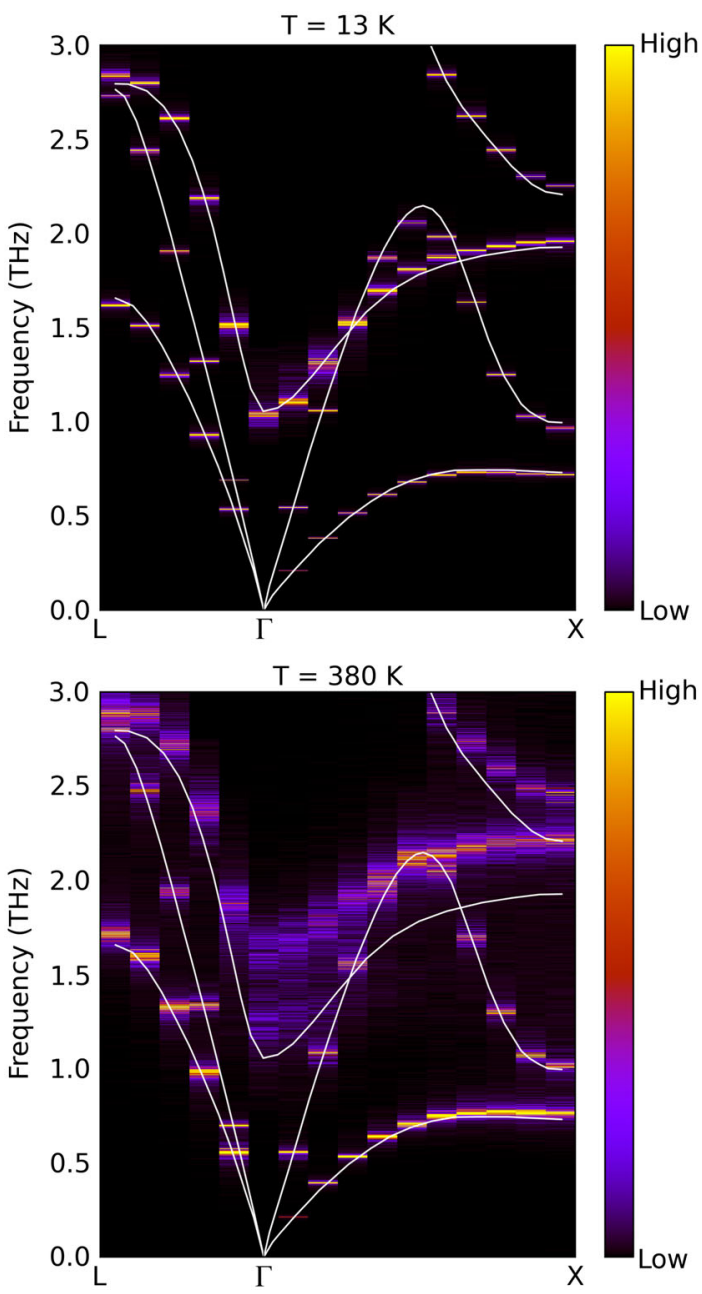

FIG. 1 (color online). The classical vibrational spectra of PbTe along the high symmetry directions in the first Brillouin zone. The white lines show the $0 \mathrm{~K}$ phonon dispersions calculated with the finite displacement method by Zhang et al. [15].

As the temperature is increased, the center of gravity of the two peaks increases and spectral weight is transferred from the lower peak to the higher peak. Between $T=230 \mathrm{~K}$ and $T=380 \mathrm{~K}$ the splitting of the peaks changes relatively little, with a value of $\approx 0.37 \mathrm{THz}$ (i.e., $\approx 1.5 \mathrm{meV}$ ). This is in good quantitative agreement with the measurements of Jensen et al. [3] (i.e., $\approx 1.6 \mathrm{meV}$ ) and Delaire et al. [2] (i.e., $\approx 1.9 \mathrm{meV}$ ). This behavior should be contrasted to the $X$ point, which behaves in a more simplistic fashion with a mild broadening and shifts of the peaks [see Fig. 2(b)].

Jensen et al. [3] proposed that the emergence of the new spectral peak is related to a local symmetry breaking in $\mathrm{PbTe}$ at elevated temperatures based on an analysis of INS measurements. This local symmetry breaking was first proposed in an earlier work where $\mathrm{Pb}$ was suggested to achieve a large average local displacement, nearly $0.2 \AA$ at room temperature, with a possible interpretation being that $\mathrm{Pb}$ fluctuates between symmetry equivalent sites [6]. Kastbjerg et al. [23] claim an even larger off-center 

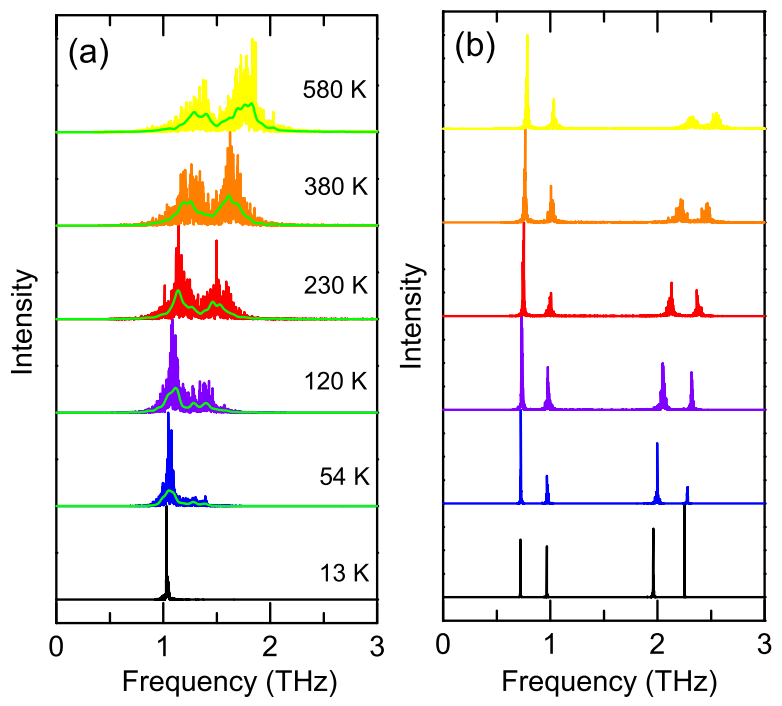

FIG. 2 (color online). The classical vibrational spectra at the zone center (a) and the zone boundary $X$ (b) at different temperatures. The solid (green) curves represent the data convolved with a Gaussian function of width $0.02 \mathrm{THz}$.

displacement of $\mathrm{Pb}$ based on synchrotron powder $\mathrm{x}$-ray diffraction. However, x-ray absorption fine structure spectroscopy experiments have been interpreted as showing no such symmetry breaking [24]. Given that our method faithfully reproduces the temperature dependence of the spectra, we can clearly answer this question with the position probability distribution function $P_{\alpha}(x, y, z)$, where $\alpha$ labels a given $\mathrm{Pb}$ or Te atom. We compute the reduced probability density $P_{r}\left(x_{1}\right)=\frac{1}{3 N_{q}} \sum_{\alpha} \int d x_{2} d x_{3}(1+$ $\left.p_{12}+p_{13}\right) P_{\alpha}\left(x_{1}, x_{2}, x_{3}\right)$, where $p_{i j}$ is the permutation operator. $P_{r}$ is symmetric and shows no indication of either ion dwelling away from zero displacement [Fig. 3(a)]. The average displacements [Fig. 3(c)] of each ion is very small (i.e., less than $0.003 \AA$ of static displacement at $380 \mathrm{~K}$ ). Furthermore, individually examining $P_{\alpha}$ shows that there is no appreciable local symmetry breaking. However, there is a large root mean square (rms) displacement which compares favorably with experiments [Fig. 3(b)]. It should also be noted that the asymmetry observed in the measured pair distribution function [6] and later reproduced in AIMD [19] are successfully captured in our simulations [25].

This novel splitting of the TO peak arises from a broadened polelike feature in the self-energy which is generated either by the third order anharmonic term or the fourth order term, and it is straightforward to separate the two. We cannot compute the spectra only using the third order term as the crystal rapidly becomes unstable. However, the spectra can be computed with the third order term set to zero, halved, and amplified 1.5 times, all while keeping the fourth order term unchanged. When $\Phi_{3}=0$, the TO mode simply shifts upward with very mild broadening (see Fig. 4). Interestingly, the peak is located close to the
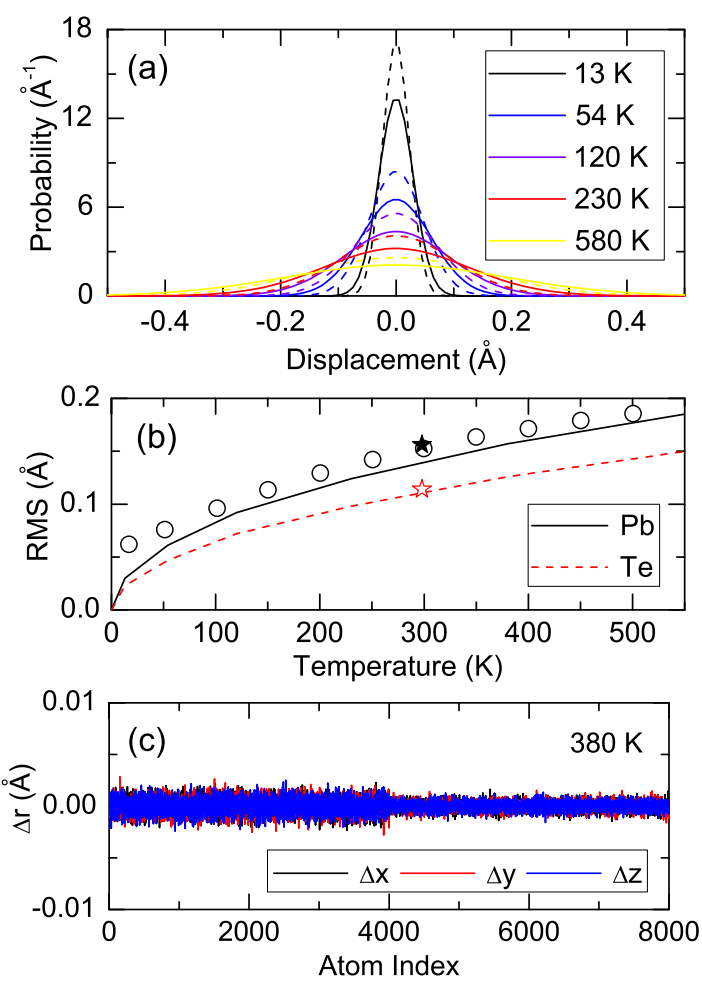

FIG. 3 (color online). (a) The reduced probability density for $\mathrm{Pb}$ (solid lines) and $\mathrm{Te}$ (dashed lines) at different temperatures. (b) The rms displacement as a function of temperature. The solid and dashed curves are computed from our MD simulations. The square root of experimental atomic displacement parameters are shown as dots [6] and stars [26]. (c) The average displacements of each of the atoms in the supercell. The first 4000 atoms are $\mathrm{Pb}$, followed by $\mathrm{Te}$.

upper peak in Fig. 2(a). In the case where the third order term is halved, the upper peak remains in a similar position but the lower peak is now closer. Finally, when the third order term is amplified 1.5 times, there is massive scattering sending large amounts of spectral weight to low energy. Therefore,

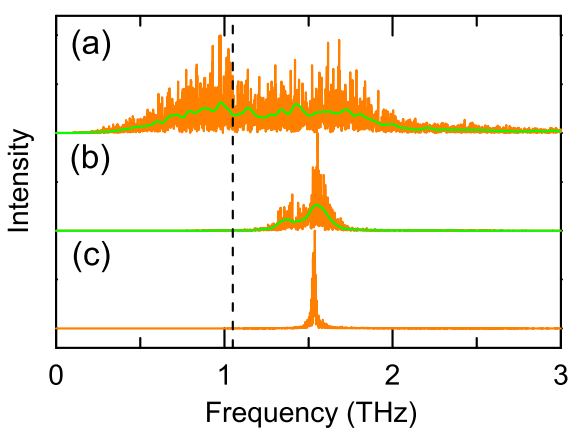

FIG. 4 (color online). The zone center classical vibrational spectra at $T=380 \mathrm{~K}$ computed with fictitious values of $\Phi_{3}$ which are multiplied by a factor of (a) 1.5 , (b) 0.5 , and (c) 0 . The solid (green) curves represent the data convolved with a Gaussian function of width $0.02 \mathrm{THz}$. The vertical dashed line is the $T=$ $0 \mathrm{~K}$ TO-mode frequency. 
we can see that the double peak is basically a confluence of both terms, and the phenomenon is rather special in that it depends on a delicate balance of two different terms.

In summary, we have demonstrated that using our slave mode expansion to compute the classical power spectrum agrees remarkably well with INS measurements. The anomalous splitting of the spectrum at the zone center is captured with our approach. We show that there are no appreciable spontaneously broken symmetries, resolving discrepancies in the experimental literature. All of this behavior can be captured with only two anharmonic parameters, which are, in essence, nearest neighbor cubic and quartic terms. Given that our work is classical, it is clear that quantum fluctuations of the phonons appear to be irrelevant to generating this spectral anomaly. Future work will be important to understanding this phenomenon in terms of traditional many-body techniques in the semiclassical limit. More broadly, our approach should be applicable to materials across the periodic table, and quantum mechanics can be incorporated with existing methods.

Y. C. and C. A. M. acknowledge funding from a Columbia RISE grant. X. A. acknowledges support from the National Science Foundation (Grant No. CMMI-1150795). We thank O. Delaire for stimulating conversations.

*Present address: Department of Mechanical Engineering, The University of Hong Kong, Pokfulam Road, Hong Kong. Corresponding author. yuechen@hku.hk

[1] S. W. Lovesey, Theory of Neutron Scattering from Condensed Matter (Oxford University Press, New York, 1986).

[2] O. Delaire, J. Ma, K. Marty, A. F. May, M. A. McGuire, M.-H. Du, D. J. Singh, A. Podlesnyak, G. Ehlers, M. D. Lumsden, and B. C. Sales, Nat. Mater. 10, 614 (2011).

[3] K. M. Ø. Jensen, E. S. Božin, C. D. Malliakas, M. B. Stone, M. D. Lumsden, M. G. Kanatzidis, S. M. Shapiro, and S. J. L. Billinge, Phys. Rev. B 86, 085313 (2012).

[4] P. Souvatzis, O. Eriksson, M. I. Katsnelson, and S. P. Rudin, Comput. Mater. Sci. 44, 888 (2009).

[5] O. Hellman, I. A. Abrikosov, and S. I. Simak, Phys. Rev. B 84, 180301(R) (2011).
[6] E. S. Božin, C. D. Malliakas, P. Souvatzis, T. Proffen, N. A. Spaldin, M. G. Kanatzidis, and S. J. L. Billinge, Science 330, 1660 (2010).

[7] D. A. Papaconstantopoulos and M. J. Mehl, J. Phys. Condens. Matter 15, R413 (2003).

[8] D. R. Bowler and T. Miyazaki, Rep. Prog. Phys. 75, 036503 (2012).

[9] J. Vandevondele, U. Borštnik, and J. Hutter, J. Chem. Theory Comput. 8, 3565 (2012).

[10] K. Esfarjani and H. T. Stokes, Phys. Rev. B 77, 144112 (2008).

[11] T. Shiga, J. Shiomi, J. Ma, O. Delaire, T. Radzynski, A. Lusakowski, K. Esfarjani, and G. Chen, Phys. Rev. B 85, 155203 (2012).

[12] X. Ai, Y. Chen, and C. A. Marianetti, Phys. Rev. B 90, 014308 (2014).

[13] J. P. Heremans, V. Jovovic, E. S. Toberer, A. Saramat, K. Kurosaki, A. Charoenphakdee, S. Yamanaka, and G. J. Snyder, Science 321, 554 (2008).

[14] R. T. Bate, D. L. Carter, and J. S. Wrobel, Phys. Rev. Lett. 25, 159 (1970).

[15] Y. Zhang, X. Z. Ke, C. F. Chen, J. Yang, and P. R. C. Kent, Phys. Rev. B 80, 024304 (2009).

[16] J. M. An, A. Subedi, and D. J. Singh, Solid State Commun. 148, 417 (2008).

[17] A. H. Romero, M. Cardona, R. K. Kremer, R. Lauck, G. Siegle, J. Serrano, and X. C. Gonze, Phys. Rev. B 78, 224302 (2008).

[18] Z. T. Tian, J. Garg, K. Esfarjani, T. Shiga, J. Shiomi, and G. Chen, Phys. Rev. B 85, 184303 (2012).

[19] Y. Zhang, X. Z. Ke, P. R. C. Kent, J. H. Yang, and C. F. Chen, Phys. Rev. Lett. 107, 175503 (2011).

[20] B. Qiu, H. Bao, G. Q. Zhang, Y. Wu, and X. L. Ruan, Comput. Mater. Sci. 53, 278 (2012).

[21] T. Chonan and S. Katayama, J. Phys. Soc. Jpn. 75, 064601 (2006).

[22] M. T. Dove, Introduction to Lattice Dynamics (Cambridge University Press, Cambridge, 1993).

[23] S. Kastbjerg, N. Bindzus, M. Søndergaard, S. Johnsen, N. Lock, M. Christensen, M. Takata, M. A. Spackman, and B. B. Iversen, Adv. Funct. Mater. 23, 5477 (2013).

[24] T. Keiber, F. Bridges, and B. C. Sales, Phys. Rev. Lett. 111, 095504 (2013).

[25] See the Supplemental Material at http://link.aps.org/ supplemental/10.1103/PhysRevLett.113.105501 for the pair distribution functions at different temperatures.

[26] P. B. Pereira, I. Sergueev, S. Gorsse, J. Dadda, E. Müller, and R. P. Hermann, Phys. Status Solidi B 250, 1300 (2013). 